\title{
Hypoxia-inducible factor-1 $\alpha$ mediates the toll-like receptor 4 signaling pathway leading to anti-tumor effects in human hepatocellular carcinoma cells under hypoxic conditions
}

\author{
XIAOYU ZHANG ${ }^{1}$, SHUCHEN LI $^{1}$, MINGRONG LI $^{1}$, HAIYING HUANG $^{1}$, JINGYUAN LI $^{1}$ and CHANGWEI ZHOU ${ }^{2}$ \\ Departments of ${ }^{1}$ Infectious Diseases and ${ }^{2}$ Orthopedics, Second Affiliated Hospital of Harbin Medical University, \\ Harbin, Heilongjiang 150086, P.R. China
}

Received March 4, 2015; Accepted March 18, 2016

DOI: $10.3892 / \mathrm{ol} .2016 .4705$

\begin{abstract}
Hypoxia-inducible factor- $1 \alpha(\mathrm{HIF}-1 \alpha)$ and toll-like receptor 4 (TLR4) are involved in numerous mechanisms of cancer biology, including cell proliferation and survival; however the interaction of the two factors under hypoxic conditions remains unclear. The present study investigated the in vitro mechanism that results in the suppression of tumor cell growth and cellular functions when HIF-1 $\alpha$ is silenced. In the present study, the human hepatocellular carcinoma HepG2 cell line was transfected with short hairpin RNA (shRNA) against HIF- $1 \alpha$ and cultured under hypoxic conditions $\left(1 \% \mathrm{O}_{2}\right.$ for $24 \mathrm{~h})$. The expression of HIF-1 $\alpha$ and various growth factors, including epidermal growth factor (EGF), hepatocyte growth factor (HGF), vascular endothelial growth factor (VEGF) and fibroblast growth factor 2 (FGF2), were examined using quantitative polymerase chain reaction and immunoblotting. Tumor growth was measured using a Cell Counting Kit-8 assay and tumor activity was measured using tumor cell invasion and migration assays. Lipopolysaccharide and TAK-242 were used to activate and inhibit TLR4, respectively, to observe the role of TLR4 in the HIF-1 $\alpha$ silenced tumor cells. The expression of TLR4 signaling pathway associates, including myeloid differentiation primary response gene 88 (MyD88), apoptosis signal-regulating kinase 1 (ASK1), p38 mitogen-activated protein kinases and HIF-1 $\alpha$, were analyzed by western blot assay. Under hypoxic conditions, silencing of HIF-1 $\alpha$ expression suppressed tumor cell growth and regulated the expression of tumor growth-associated genes, including EGF, HGF, VEGF and FG2. Suppression of tumor cell invasion and migration was also observed in the HIF-1 $\alpha$ silenced HepG 2 cell
\end{abstract}

Correspondence to: Professor Changwei Zhou, Department of Orthopedics, Second Affiliated Hospital of Harbin Medical University, 246 Xuefu Road, Nangang, Harbin, Heilongjiang 150086, P.R. China

E-mail: changweizhou2014@163.com

Key words: hypoxia inducible factor- $1 \alpha$, toll-like receptor 4 signaling pathway, HepG2 cell line, anti-tumor effects line. In addition, TLR4 was identified to be involved in HIF-1 $\alpha$ and MyD88 accumulation, and activation of ASK1 and p38 were demonstrated to be critical for TLR4-mediated HIF-1 $\alpha$ pathway. In conclusion, silencing of HIF-1 $\alpha$ expression may induce anti-tumor effects under hypoxic conditions in HepG2 cells via the TLR4 mediated pathway, suggesting that the HIF-1 $\alpha /$ TLR4 signaling cohort may act as a novel therapeutic target for the treatment of hepatocellular cancer.

\section{Introduction}

Worldwide, hepatocellular cancer (HCC) is the sixth most common malignant tumor and the third leading cause of cancer-associated mortality $(1,2)$. One of the most well-known characteristics of the tumor microenvironment identified in HCC is tumor hypoxia (3). HCC originates from cirrhosis induced by chronic liver injury; chronic injury causes fibrogenesis, which destroys the normal liver blood system and leads to a shortage of blood circulation, resulting in hypoxia. In addition, the high proliferation of tumor cells induces local hypoxia within the microenvironment of HCC (4).

The cellular response to hypoxia is mediated by the hypoxia-inducible factors (HIFs) family of transcription factors (5). Hypoxia induced factor-1 (HIF-1) is the major transcription factor of this family, and is composed of two subunits: Oxygen-sensitive HIF-1 $\alpha$ and aryl hydrocarbon receptor nuclear translocator (HIF-1 $\beta$ ). Under low oxygen tension, HIF-1 $\alpha$ upregulates various hypoxia inducible genes through dimerization with HIF-1 $\beta$, and binds to the hypoxia-responsive subunit in the promoter of target genes (6).

Hypoxia and inflammation are closely associated and are important in a variety of pathological situations. Hypoxia elicits tissue inflammation; during acute organ ischemia, including intestinal or hepatic ischemia, the ischemic organ becomes severely inflamed (7). However, alterations in the signaling pathway involved in this pathological process remain unknown.

Toll-like receptor 4 (TLR4) is a mammalian pattern recognition receptor, which recognizes lipopolysaccharide (LPS) as a ligand (8). TLR4 is closely involved in the development and progression of various inflammatory diseases, such as inflammatory bowel disease and atherosclerosis (9-11). A previous study has demonstrated that 
downstream signaling of TLR4 leads to an accumulation of HIF-1 $\alpha$, which is important for TLR4-dependent expression of proinflammatory cytokines (12). Certain studies have also demonstrated that bacteria or LPS-induced HIF-1 $\alpha$ accumulation is TLR4 dependent in immune cells $(12,13)$. In human myeloid monocytic leukemia THP-1 cells, LPS-induced TLR4 signaling triggered crosstalk between HIF-1 $\alpha$ and apoptosis signal-regulating kinase 1 (ASK1) (14). ASK1 contributes to the stabilization of HIF- $1 \alpha$ possibly via the activation of p38 mitogen-activated protein kinase (MAPK) (14). ASK1 and p38 are important downstream signaling molecules of the TLR4 signaling pathway (15).

The present study demonstrated the effects of silencing HIF-1 $\alpha$ expression in human hepatocellular carcinoma HepG 2 cells, and demonstrated that HIF- $1 \alpha$ silencing leads to the suppression of tumor cell growth, invasion and migration via the TLR4 signaling pathway.

\section{Materials and methods}

Materials. Dulbecco's modified Eagle's medium (DMEM), fetal bovine serum (FBS), penicillin/streptomycin, Basement Membrane Matrix and phosphate-buffered saline (PBS) were obtained from Invitrogen ${ }^{\mathrm{TM}}$ (Thermo Fisher Scientific, Inc., Waltham, MA, USA). Resatorvid (TAK-242) was purchased from MedChem Express (Monmouth Junction, NJ, USA). LPS derived from Escherichia coli J5 (catalog no., L5014) was obtained from Sigma-Aldrich (St. Louis, MO, USA) and ECL $^{\circledR}$ Plus Western Blotting Detection System was from Bio-Rad Laboratories, Inc. (Hercules, CA, USA). Antibodies against HIF-1 $\alpha$, epidermal growth factor (EGF), hepatocyte growth factor (HGF), vascular endothelial growth factor (VEGF), fibroblast growth factor 2 (FGF2), TLR4, caspase-3, p-ASK1, p-p38 and myeloid differentiation primary response gene 88 (MyD88) were purchased from Cell Signaling Technology, Inc. (Danvers, MA, USA). The Cell Counting kit- 8 (CCK-8) was from Dojindo Molecular Technologies, Inc. (Kumamoto, Japan). Reagents for quantitative polymerase chain reaction (qPCR) analysis, including reverse transcriptase kit from Promega (Madison, WI, USA) and Thunderbird SYBR qPCR Mix from Toyobo, Co., Ltd., (Osaka, Japan). Nuclear extraction kits were purchased from Takara Biotechnology Co., Ltd. (Dalian, China).

Cell culture. HepG2 cells were purchased from American Type Culture Collection (Manassas, VA, USA) and cultured at $37^{\circ} \mathrm{C}$ with $5 \% \mathrm{CO}_{2}$ in DMEM supplemented with $10 \% \mathrm{FBS}$, $4.5 \mathrm{~g} / 1$ glucose, $2 \mathrm{mM}$ L-glutamine (Thermo Fisher Scientific, Inc.), $100 \mathrm{U} / \mathrm{ml}$ penicillin and $100 \mu \mathrm{g} / \mathrm{ml}$ streptomycin.

Short hairpin RNA (shRNA) and transfection. shRNA targeting the human HIF-1 $\alpha$ gene and scramble control sequences were purchased from Santa Cruz Biotechnology, Inc. (Dallas, TX, USA). The targeted sequence of sh-HIF-1 $\alpha$ was 5'-CCCTAATTGAGTCAAACTTGAGCTTTCAAGTTT GACTCAATTAGGGAAAA-3', and the targeted sequence of the negative control shNC was 5'-AGGGAAAACCCTAAT TGAGTCAAACTTGAGCTTTCAAGTTTGACTCAATT-3'. Human HIF-1 $\alpha$ shRNA was inserted into the recombinant plasmid pGPU6 (Shanghai GenePharma Co., Ltd., Shanghai,
China). For HIF-1 $\alpha$ gene silencing, HepG2 cells were transfected with pGPU6-shHIF-1 $\alpha$ or shNC plasmids using Lipofectamine 2000 (Invitrogen; Thermo Fisher, Scientific, Inc.). The efficiency of transfection was evaluated by western blotting with antibodies against HIF-1 $\alpha$.

Following transfection, the cells were maintained at normoxic conditions (95\% air, 5\% $\mathrm{CO}_{2}$ ) for $24 \mathrm{~h}$, and the cells were subsequently placed in Krebs-Ringer Bicarbonate buffer $\left(115 \mathrm{mM} \mathrm{NaCl}, 4.7 \mathrm{mM} \mathrm{KCl}, 2.5 \mathrm{mM} \mathrm{CaCl}_{2}, 1.2 \mathrm{mM}\right.$

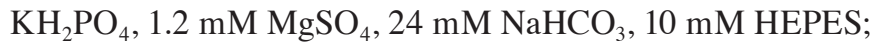
$\mathrm{pH}$ 7.4), followed by culturing at $95 \% \mathrm{~N}_{2}$ and $5 \% \mathrm{CO}_{2}$ in a hypoxic environment for $24 \mathrm{~h}$. In total, $12 \mathrm{~h}$ prior to hypoxia, cells were exposed to LPS $(1 \mu \mathrm{g} / \mathrm{ml})$ or TAK-242 $(1 \mu \mathrm{M})$. The LPS and TAK-242 remained in the culture medium and the cells were washed three times with PBS and then placed into DMEM medium with $10 \%$ FBS.

Cell viability and cell growth assays. HepG2 cells were exposed to indicated hypoxia conditions at a density of $1 \times 10^{4}$ cells/well for 24,48 and $72 \mathrm{~h}$. The number of viable cells was counted by trypan blue dye (Beyotime Institute of Biotechnology, Shanghai, China) exclusion using a hemocytometer.

The viability of the HepG2 cells was measured using the CCK-8 assay according to the manufacturer's protocol. Briefly, HepG2 cells were seeded in a 96-well plate (NEST Biotechnology, Suzhou, China) at $1 \times 10^{4}$ cells/well, incubated at $37^{\circ} \mathrm{C}$ for $24 \mathrm{~h}$, then subjected to a hypoxic environment for $24 \mathrm{~h}$, after which CCK-8 solution was added for another $4 \mathrm{~h}$ incubation. Optical density was measured using a microplate reader (Multiskan ${ }^{\mathrm{TM}}$ MK3; Thermo Fisher Scientific, Inc.) at $450 \mathrm{~nm}$.

Reverse transcription qPCR. Total RNA was extracted from HepG2 cells using TRIzol Reagent (Invitrogen; Thermo Fisher Scientific, Inc.). RNA concentration was determined by UV spectrophotometry (NanoDrop 2000; Thermo Fisher Scientific, Inc.). cDNA was synthesized from $1 \mu \mathrm{g}$ of RNA (positive control) using reverse transcriptase kit from Promega. The protocol was repeated using $\mathrm{ddH}_{2} \mathrm{O}$ instead of RNA, as the negative control. qPCR was performed using Thunderbird SYBR qPCR Mix from Toyobo, Co., Ltd. The primer sequences were designed by PrimerBank (https://pga.mgh.harvard.edu/primerbank) and synthesized by GeneScript (Nanjing, China) as follows: HIF-1 $\alpha$, forward 5'-ACTTGGCAACCTTGGATTGGA-3' and reverse 5'-ATC TCCGTCCCTCAACCTCT-3' (190 bp); EGF, forward 5'-AGA GGGAGAGGATGCCACAT-3' and reverse 5'-GGGGTG GAGTAGAGTCAAGA-3' (206 bp); HGF, forward 5'-ACA GCTTTTTGCCTTCGAGC-3' and reverse 5'-GCAAGAATT TGTGCCGGTGT-3' (261 bp); VEGF, forward 5'-TCACCA AGGCCAGCACATAG-3' and reverse 5'-GAGGCTCCA GGGCATTAGAC-3' (202 bp); FGF2, forward 5'-TCCACC TATAATTGGTCAAAGTGGT-3' and reverse 5'-CATCAG TTACCAGCTCCCCC-3' (121 bp); tumor necrosis factor (TNF)- $\alpha$, forward 5'-CTGGGCAGGTCTACTTTGGG-3' and reverse 5'-CTGGAGGCCCCAGTTTGAAT-3' (272 bp); interleukin (IL)-6, forward: 5'-TGCAATAACCACCCCTGACC-3' and reverse 5'-GTGCCCATGCTACATTTGCC-3' (163 bp). qPCR was performed on a StepOnePlus ${ }^{\mathrm{TM}}$ Real-time PCR 
$\mathbf{A}$

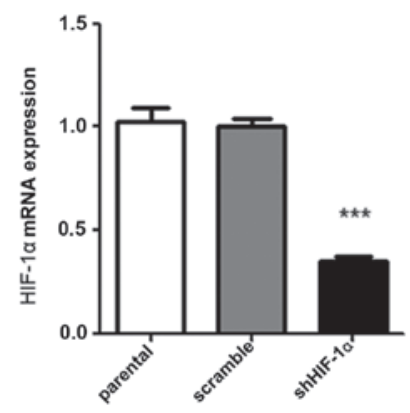

B

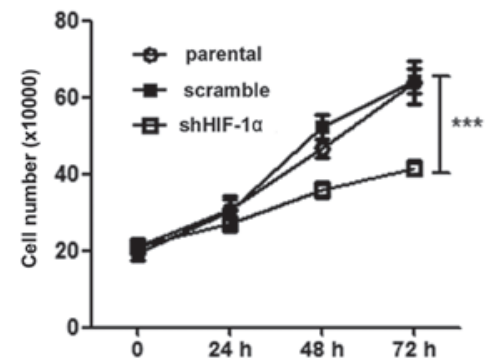

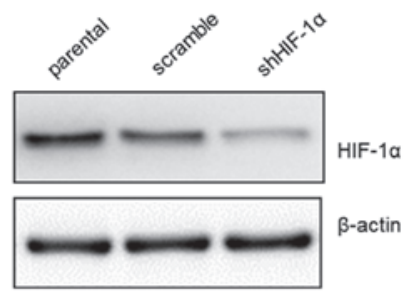

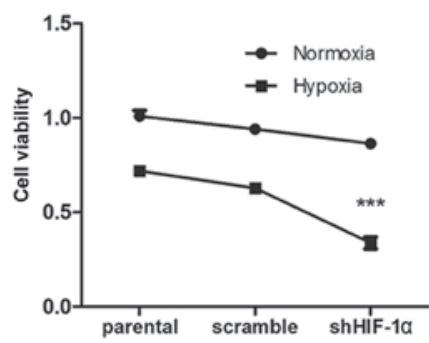

Figure 1. Suppression of tumor cell growth through silencing of HIF-1 $\alpha$. Human hepatocellular carcinoma HepG2 cells were transfected with shRNAs against HIF-1 $\alpha$ or scramble sequences for $48 \mathrm{~h}$, followed by exposure to hypoxic conditions $\left(1 \% \mathrm{O}_{2}\right)$ for various amounts of time. (A) mRNA was extracted using TRIzol for quantitative polymerase chain reaction and $30 \mathrm{mg}$ total lysate was harvested with radioimmunoprecipitation assay buffer for western blot analysis, to detect the mRNA and protein levels of HIF-1 $\alpha$ following transfection. (B) Tumor cell growth following silencing of HIF-1 $\alpha$ was measured using a Cell Counting kit-8. Tumor cells were susceptible to growth retardation under hypoxic conditions following transfection with shHIF-1 $\alpha$. Transfection of shHIF-1 $\alpha$ suppressed cell growth when maintained at hypoxic conditions compare with parental and scramble sh groups. Data are presented as the mean \pm standard error of the mean of three independent experiments. ${ }^{* * *} \mathrm{P}<0.001$ vs. parental group. HIF-1 $\alpha$, hypoxia induced factor- $1 \alpha$; sh, short hairpin.

System (Applied Biosystems ${ }^{\mathrm{TM}}$; Thermo Fisher Scientific, Inc.) with the following cycle: $95^{\circ} \mathrm{C}$ for $1 \mathrm{~min}$, followed by $95^{\circ} \mathrm{C}$ for $15 \mathrm{sec}, 58^{\circ} \mathrm{C}$ for $30 \mathrm{sec}$, and $72^{\circ} \mathrm{C}$ for $30 \mathrm{sec}$ for 40 cycles. Glyceraldehyde 3-phosphate dehydrogenase (GAPDH; forward 5'-GATCCCGCTAACATCAAATG-3' and reverse 5'-GAGGGAGTTGTCATATTTCTC-3') expression was used as an internal control. $2^{-\triangle \Delta C q}$ was calculated for every sample and the mRNA expression levels were indicated with $2^{-\Delta \Delta C q}$ and normalized to GAPDH (16). The experiments were repeated three times independently and positive (cDNA from HepG2 cells) and negative (no cDNA) controls were included.

Tumor cell migration assay. The effects of HIF- $1 \alpha$ knockdown on tumor cell migration was investigated in HepG2 cells grown in serum-free DMEM. Briefly, HepG2 cells were seeded into a 6-well plate (NEST Biotechnology) and reach $90 \%$ confluence. A single scratch was created on confluent monolayers using a micropipette tip $(1 \mathrm{~mm})$. Subsequently, wounded monolayers were washed with PBS to remove cell debris, supplemented with serum-free DMEM and subjected to hypoxic conditions for $24 \mathrm{~h}$. The median distance of migrating cells to the wound was determined under an inverted microscope (IX81; Olympus Corporation, Tokyo, Japan) at 0 and $24 \mathrm{~h}$.

Tumor cell invasion assay. The invasiveness of tumor cells was assessed in vitro using a Transwell chamber, as previously described (17). In brief, cells were seeded in 24-well Transwell plates (EMD Millipore, Billerica, MA, USA) in $10 \%$ FBS medium at $1 \times 10^{5}$ cells/well. After $24 \mathrm{~h}$, the medium was changed to free-serum DMEM and the cells were subjected to hypoxia for $24 \mathrm{~h}$, while $10 \%$ FBS DMEM was added to the lower chamber. Non-adherent cells were washed away with PBS and adherent cells were fixed in ethanol. After staining with $0.1 \%$ crystal violet, pictures were taken using a microscope (IX81; Olympus, Tokyo, Japan).

Western blot analysis. Total cell lysate was extracted from HepG2 cells using radioimmunoprecipitation assay lysis buffer (Cell Signaling Technology, Inc.), according to the manufacturer's protocol, resolved by $10 \%$ sodium dodecyl sulfate-polyacrylamide gel electrophoresis and transferred to nitrocellulose membranes. The membranes were blocked with 5\% non-fat dry milk in Tris-buffered saline (TBS; Solarbio, Beijing, China) for $2 \mathrm{~h}$ at room temperature. Thereafter, the membranes were incubated with primary antibodies against HIF-1 $\alpha$ (dilution, 1:1,000; rabbit polyclonal; catalog no., ab113642), phospho (p)-ASK1 (dilution, 1:1,000; rabbit polyclonal; catalog no., 3764), p-p38 MAPK (dilution, 1:1,000; rabbit polyclonal; catalog no., 9211), caspase-3 (dilution, 1:1,000; rabbit monoclonal; catalog no., 9665) (Cell Signaling Technology, Inc.), TLR4 (dilution, 1:1,000; rabbit polyclonal; catalog no., ab47839), MyD88 (dilution, 1:1,000; rabbit polyclonal; catalog no., ab2064), EGF (dilution, 1:1,000; rabbit polyclonal; catalog no., ab9695), FGF2 (dilution, 1:1,000; rabbit polyclonal; catalog no., ab126861), VEGF (dilution, 1:1,000; rabbit polyclonal; catalog no., ab46154) and HGF (dilution, 1:1,000; rabbit polyclonal; catalog no., ab83760) (Abcam, Cambridge, MA, USA) at $4^{\circ} \mathrm{C}$ overnight. After being washed three times for $10 \mathrm{~min}$ in TBS with $0.05 \%$ Tween 20 (Solarbio), the membranes were incubated with horseradish peroxidase-conjugated anti-rabbit secondary antibody (dilution, 1:5,000; catalog no., 7074; Cell Signaling Technology, Inc.) or anti-mouse secondary 

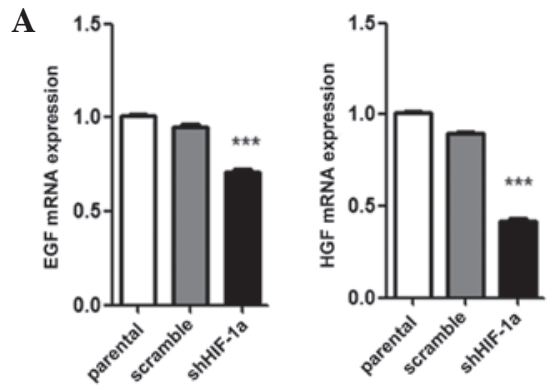

B
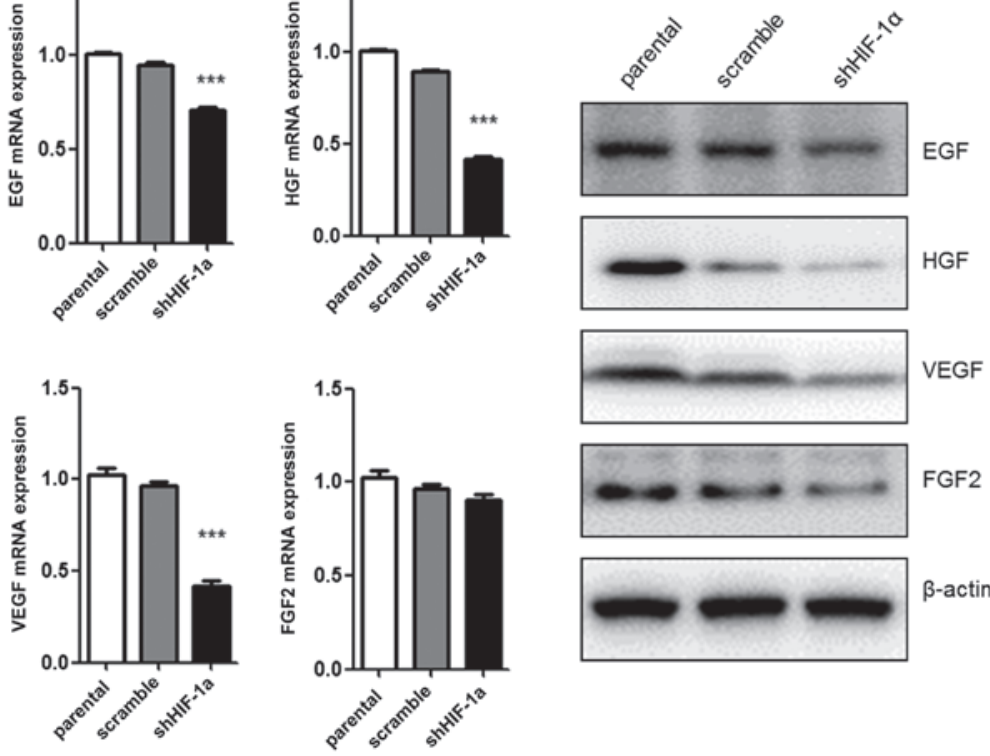

Figure 2. Silencing of HIF-1 $\alpha$ affects protein expression of tumor growth-associated genes in human hepatocellular carcinoma HepG2 cells. Decreased expression of EGF, HGF, VEGF and FGF2 mRNA and protein levels by silencing of HIF-1 $\alpha$ were confirmed by (A) quantitative polymerase chain reaction and (B) western blot analysis. mRNA was extracted with TRIzol for quantitative polymerase chain reaction and $30 \mathrm{mg}$ total lysate was harvested with radioimmunoprecipitation assay buffer for western blot analysis. Data are presented as the mean \pm standard error of the mean of three independent experiments. ${ }_{* * * *} \mathrm{P}<0.001$ vs. parental group. EGF, epidermal growth factor; HGF, hepatocyte growth factor; VEGF, vascular endothelial growth factor; FGF2, fibroblast grow factor-2; HIF-1 $\alpha$, hypoxia induced factor-1 $\alpha$; sh, short hairpin.

A
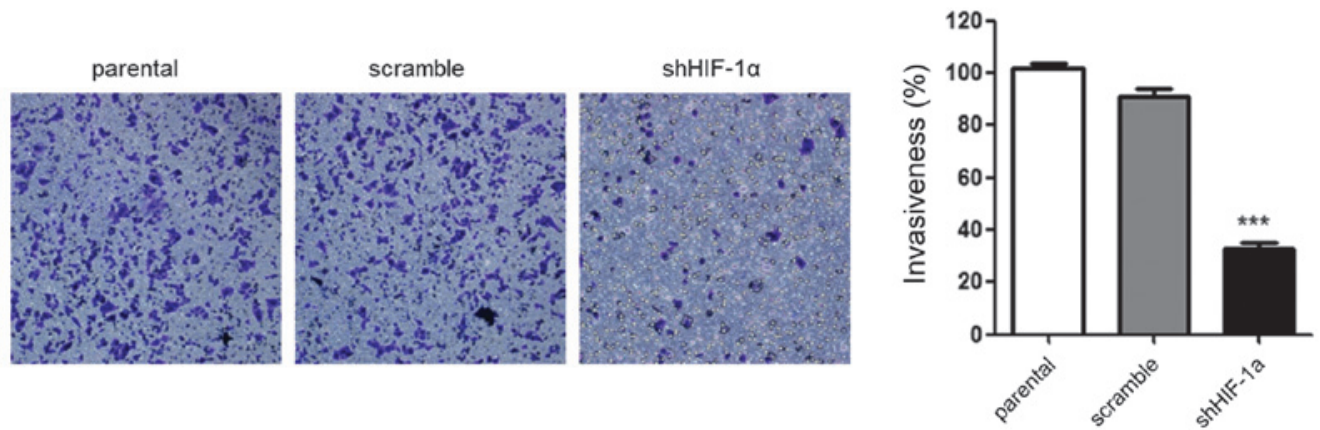

B
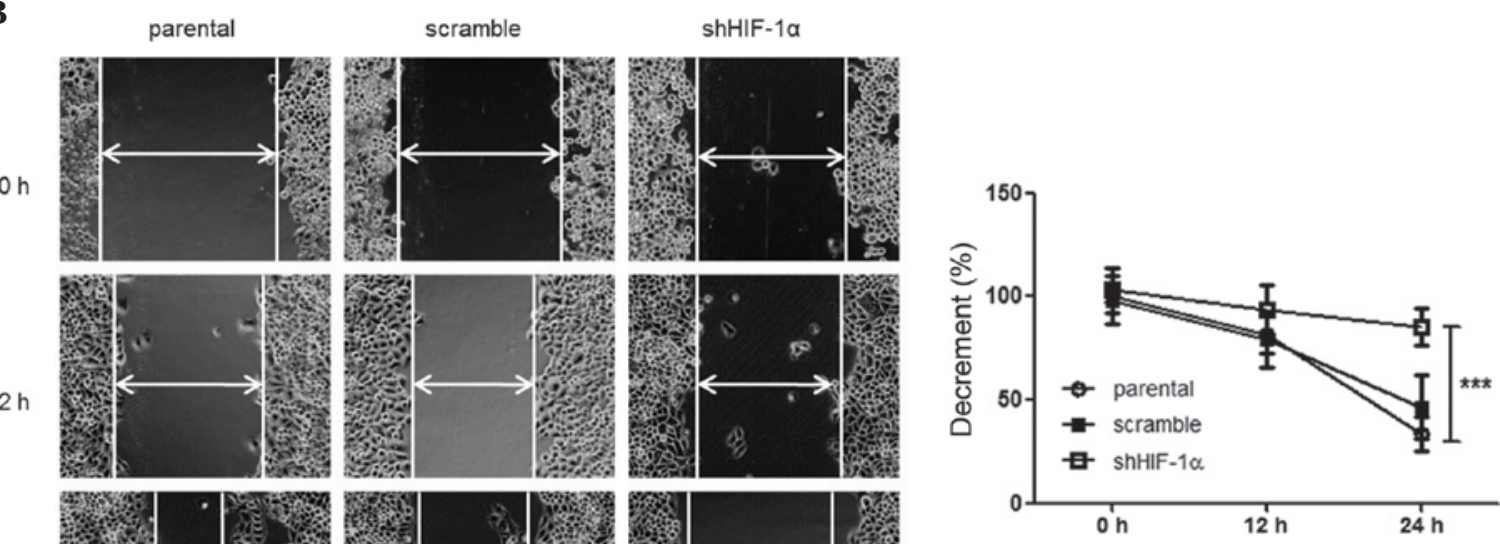

$24 \mathrm{~h}$
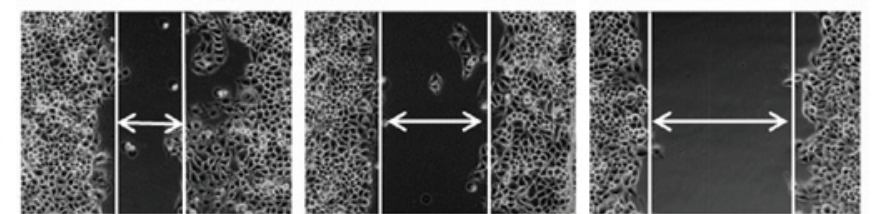

Figure 3. HIF-1 $\alpha$ silencing suppresses tumor cell invasive and migration ability in hypoxic conditions. (A) Human hepatocellular carcinoma HepG2 cells were subjected to hypoxic conditions for $24 \mathrm{~h}$. Compared with parental cells, the invasive ability of the tumor cells was significantly reduced in HepG2 cells transfected with shHIF-1 $\alpha$, as demonstrated by Transwell assay. (B) Compared with parental cells, the migration ability of the HIF-1 $\alpha$ silenced HepG2 cells was decreased, as confirmed by a wound healing assay under hypoxic conditions for 12 and $24 \mathrm{~h}$. Data are presented as the mean \pm standard error of the mean. ${ }^{* * * *} \mathrm{P}<0.001$ vs. parental group. HIF-1 $\alpha$, hypoxia induced factor-1 $\alpha$; sh, short hairpin. 
A

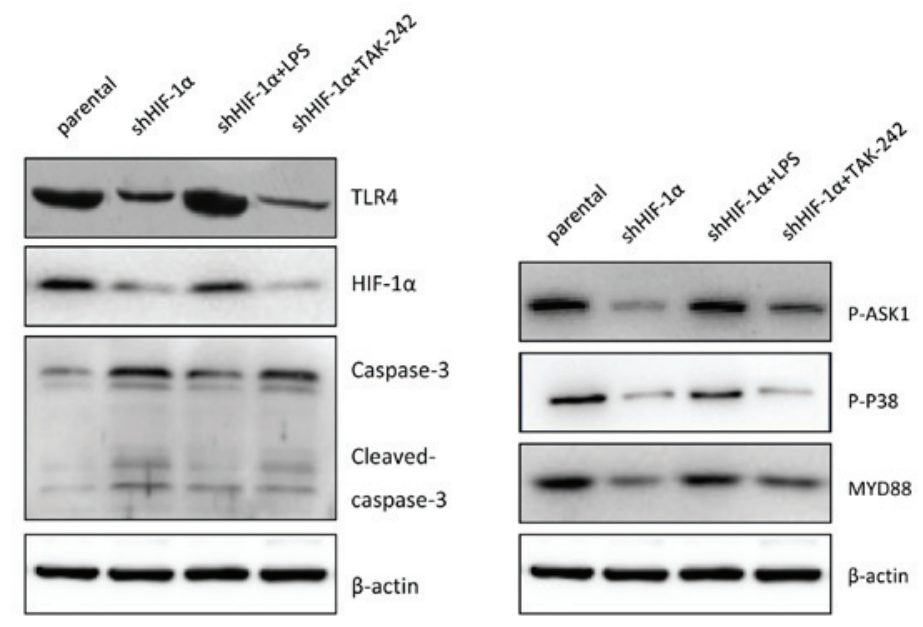

B
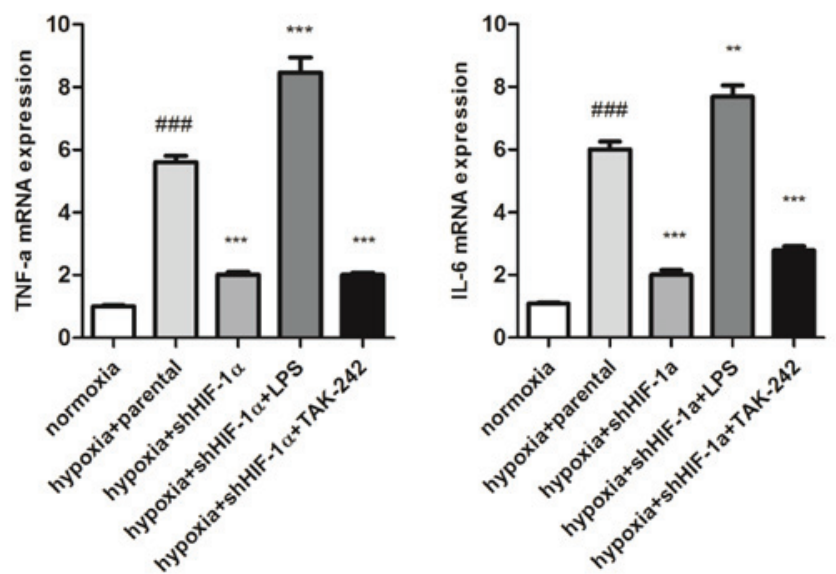

Figure 4. Involvement of TLR4 signaling pathway in HIF-1 $\alpha$ silencing affects inflammatory and apoptosis under hypoxic conditions. (A) In total, $30 \mathrm{mg}$ total lysate was harvested with radioimmunoprecipitation assay buffer to detect TLR4, HIF-1 $\alpha$, caspase-3, MyD88, p-ASK1 and p-p38 protein expression by western blot analysis in human hepatocellular carcinoma HepG2 cells. The expression of TLR4, HIF-1 $\alpha$, MyD88, p-ASK1 NS and p-p38 in shHIF-1 $\alpha$ transfected cells was significantly higher compared with the parental cells. $1 \mu \mathrm{g} / \mathrm{ml}$ LPS and $1 \mu \mathrm{M}$ TAK-242 markedly mediated the expression of these proteins compared with the shHIF-1 $\alpha$ transfected cells. (B) Inflammatory cytokines (TNF- $\alpha$ and IL-6) mRNA expression levels were detected by quantitative polymerase chain reaction. Data are presented as the mean \pm standard error of the mean of three independent experiments. ${ }^{\# \#} \mathrm{P}<0.001$ vs. normoxia group; ${ }^{* * *} \mathrm{P}<0.001$ vs. hypox ia + parental group. HIF-1 $\alpha$, hypoxia induced factor-1 $\alpha$; sh, short hairpin; TLR4, toll-like receptor 4; LPS, lipopolysaccharide; p-, phospho-; ASK1, apoptosis signal-regulating kinase 1; MyD88, myeloid differentiation primary response gene 88; TNF- $\alpha$, tumor necrosis factor- $\alpha$; IL-6, interleukin-6.

antibody (dilution, 1:5,000; catalog no., 7076; Cell Signaling Technology, Inc.) for $1 \mathrm{~h}$ at room temperature. Specific bands were detected using the Pierce ECL Western Blotting Substrate (Thermo Fisher Scientific, Inc.). $\beta$-actin (dilution, 1:3,000; rabbit polyclonal; catalog no., 4967; Cell Signaling Technology, Inc.) was used as an internal control.

Statistical analysis. Results are presented as the mean \pm standard error of the mean. Independent t-tests were performed to compare the difference of the means between control and experiment groups. All statistical analysis was performed using SPSS version 12.0 (SPSS, Inc., Chicago, IL, USA). $\mathrm{P}<0.05$ was considered to indicate a statistically significant difference.

\section{Results}

Silencing of HIF-1 $\alpha$ suppresses tumor cell growth. After transfection of HepG2 cells with shHIF-1 $\alpha$, tumor cell growth was assessed using a CCK- 8 assay. In total, $48 \mathrm{~h}$ subsequent to transfection, tumor cell growth was significantly suppressed compared with the control group $(\mathrm{P}=0.012)$ (Fig. 1). The negative effect of HIF-1 $\alpha$ silencing on tumor cell growth was more prominent under hypoxic conditions. These findings were consistent with previous studies (6). Silencing of HIF-1 $\alpha$ may exhibit a suppressive tumor effect under hypoxic conditions, and is possibly mediated by inhibition of several cell proliferation genes.

Silencing of HIF-1a affects the expression of tumor growth-associated genes. Since tumor cell proliferation was inhibited by HIF-1 $\alpha$ silencing, the present study evaluated the mRNA and protein levels of several growth factors involved in tumor cell growth. EGF, FGF, VEGF and HGF mRNA expression levels were quantified by qPCR (Fig. 2A). Under hypoxic conditions, cells transfected with shHIF-1 $\alpha$ exhibited low levels of EGF ( $\mathrm{P}=0.035)$, FGF $(\mathrm{P}=0.027)$ and VEGF $(\mathrm{P}=0.016)$ expression compared with parental cells. However, the mRNA level of FGF2 expression was not affected by HIF-1 $\alpha$ silencing. The protein expression levels of these growth factors were detected by western blot analysis, and demonstrated the same results as the mRNA analysis 
(Fig. 2B). Collectively, these data suggest that HIF-1 $\alpha$ expression regulates the expression of tumor growth-associated factors, including EGF, HGF and VEGF, but not FGF2, under hypoxic conditions.

HIF-1 $\alpha$-silencing suppresses tumor cell invasiveness and motility. Since tumor cells may mobilize and invade into adjacent and distant regions, the effect of HIF-1 $\alpha$ silencing on the ability of HepG2 cells to migrate and invade was investigated using wound healing and Transwell assays. The effect of HIF-1 $\alpha$ silencing on the invasiveness of tumor cells was investigated using Matrigel-coated Transwell chambers. There was a decreased number of migrated shHIF-1 $\alpha$-tranfected HepG2 cells compared with parental cells, demonstrating that there was a suppression of the invasive ability of the tumor cells $(\mathrm{P}=0.027)$ (Fig. 3A). Similarly, as compared with the parental cells, there was a marked decrease in the migration of shHIF-1 $\alpha$-transfected HepG2 cells in the wound healing assay ( $\mathrm{P}=0.024)$ (Fig. 3B). Collectively, these data suggest that silencing of HIF-1 $\alpha$ affects the invasiveness and migration of HepG2 cells under hypoxic conditions.

HIF-1 $\alpha$-silencing induces apoptosis in a TLR4-dependent fashion. Subsequently, the present study evaluated the alterations in HIF-1 $\alpha$ expression following treatment of the HepG2 cells with LPS and TAK-242, which activates or inhibits TLR4 signaling, respectively. The present study demonstrated that LPS enhanced mRNA and protein accumulation of HIF-1 $\alpha$, and TAK-242 substantially inhibited the expression of HIF-1 $\alpha$ compared with the control group under hypoxic conditions (Fig. 4A).

To understand the role of TLR4 signaling downstream in TLR-4 mediated HIF-1 $\alpha$ accumulation under hypoxic conditions, MyD88, p-ASK1 and p-p38 protein expression was analyzed using western blot analysis. The results demonstrated that MyD88, p-ASK1 and p-p38 were significantly decreased in HIF-1 $\alpha$-silenced cells compared with parental cells. LPS markedly enhanced and TAK-242 markedly reduced the expression of MyD88, p-ASK1 and p-p38 (Fig. 4A).

Compared with the hypoxia parental cells, the inflammatory response and cell apoptosis were markedly attenuated by silencing HIF-1 $\alpha$, which was demonstrated by the expression of inflammatory cytokines (TNF- $\alpha$ and IL-6; $\mathrm{P}=0.036$ and $\mathrm{P}=0.027$, respectively) and cleaved caspase- 3 expression, which is an active apoptosis regulating molecule (Fig. 4A and B). These results suggest that HIF-1 $\alpha$ significantly affects cell inflammation and apoptosis in hypoxic conditions.

\section{Discussion}

Hypoxia is known as one of the basic hallmarks of solid tumors (18). HIF-1 is important in the cellular response to tumor hypoxia and imposes the biggest challenge in oncotherapy $(19,20)$. In the tumor, HIF-1 $\alpha$ is an important inducer of cell proliferation, metastasis, neovascularization and survival $(21,22)$, and is regulated by oxygen concentration. Under hypoxic conditions, HIF-1 $\alpha$ is concentrated in the cytoplasm, while in normoxia, HIF-1 $\alpha$ proteins are quickly degraded (23). TLR4 is one of the most physiologically important TLRs, and recognizes LPS as a ligand. It induces activation of downstream signaling networks that initiate innate immune signaling cascades and pro-inflammatory responses (24). The present study investigated the functions of HIF-1 $\alpha$ on tumors under hypoxic conditions using shHIF-1 $\alpha$ to silence the mRNA and protein expression of HIF-1 $\alpha$ in HepG2 cells. The present study demonstrated that TLR4 expression was mediated by HIF- $1 \alpha$.

In the present study, HepG2 cells were transfected with shRNA to HIF-1 $\alpha$ and the cells were subjected to hypoxic conditions. As previously reported, the effect of this inhibition on tumor growth was observed using CCK-8 assay and a cell viability assay (6). Subsequently, based on the observation that tumor growth was inhibited in these cells, the expression of growth factors, including EGF, HGF, VEGF and FGF2, which are associated with tumor growth, were assessed using qPCR and western blot analysis. EGF is a growth factor that stimulates cell proliferation, growth and differentiation by binding to its receptor (25). HGF regulates cell growth and motility by activating a tyrosine kinase signaling cascade, while VEGF is a signaling protein produced by cells which stimulates neovasculogenesis and angiogenesis (26). FGF2 is associated with the regulation of tumor angiogenesis and metastasis (27). The present study revealed that silencing HIF-1 $\alpha$ in HepG2 cells downregulates the mRNA and protein expression of these four growth factors, leading to anti-tumor effects, which is consistent with previous findings (28). The influence of hypoxia and HIF-1 $\alpha$ knockdown in HepG2 cells was evaluated using wound healing and tumor cell invasion assays. The present study demonstrated that when HIF-1 $\alpha$ expression was inhibited cell migration was suppressed and the invasive abilities of the cells were decreased.

TLR4 downstream signaling involves recruitment of adapter proteins, including MyD88 (29). MyD88-dependent signaling is associated with HIF-1 $\alpha$ activation and MyD88 is required to stabilize HIF-1 $\alpha$ under normoxic conditions following stimulation with LPS (30). The present results indicate that MyD88-dependent TLR4 signaling is involved in HIF-1 $\alpha$ activation under hypoxic conditions and affect apoptosis-associated caspase- 3 activation. ASK1 and p38 are considered to be involved in the activation of TLR4. ASK1 is an evolutionarily conserved MAP3-kinase, and an active form of the kinase interacts with the TNF receptor associated factor 6 forming a catalytically active complex, which activates p38 MAPK (15). To investigate the role of ASK1-p38 in TLR4-mediated HIF-1 $\alpha$ signaling under hypoxia, p-ASK1, p-p38 and HIF-1 $\alpha$ levels were assessed in the present study. The present results revealed that expression of these proteins in the parental hypoxia group of cells was significantly higher compared with the shHIF-1 $\alpha$ group. Furthermore, LPS and TAK-242 treatment markedly mediated the expression of p-ASK1,p-p38 and cleaved caspase- 3 in shHIF-1 $\alpha$ HepG2 cells compared with the parental/hypoxia and shHIF-1 $\alpha$ groups that were not treated with LPS and TAK-242. Therefore, the present authors suggest that TLR4 signaling leads to an accumulation of HIF-1 $\alpha$ by ASK1-activated p38 under hypoxic conditions.

In conclusion, HIF- $1 \alpha$ is known to have a critical role in tumor development, since it promotes energy metabolism, proinflammatory responses and apoptosis. In the present study, apoptosis-associated factors and inflammatory cytokine TNF- $\alpha$ amd IL- 6 mRNA release were detected using western 
blot analysis and qPCR. The present results revealed that inhibition of HIF-1 $\alpha$ activity leads to a markedly increased activation of caspase-3, while LPS and TAK-242 treatment mediate that activation. LPS-activated TLR4 may promote the production of inflammatory cytokines in HepG2 cells, and HIF-1 $\alpha$ silencing and TAK-242 treatment may decrease the release of these cytokines. The present results support the hypothesis of the present authors that HIF-1 $\alpha$ may mediate TLR4 activation dependent proinflammatory cytokine release and apoptosis of tumor cells, suggesting that the HIF-1 $\alpha /$ TLR4 signaling cohort may act as a novel therapeutic target for the treatment of HCC.

\section{References}

1. Ferenci P, Fried M, Labrecque D, Bruix J, Sherman M, Omata M, Heathcote J, Piratsivuth T, Kew M, Otegbayo JA, et al: World gastroenterology organisation global guideline. Hepatocellular carcinoma (hcc): A global perspective. J Gastrointestin Liver Dis 19: 311-317, 2010.

2. Nordenstedt H, White DL and El-Serag HB: The changing pattern of epidemiology in hepatocellular carcinoma. Dig Liver Dis 42 (Suppl 3): S206-S214, 2010.

3. Greer SN, Metcalf JL, Wang Y and Ohh M: The updated biology of hypoxia-inducible factor. EMBO J 31: 2448-2460, 2012.

4. Wu XZ, Xie GR and Chen D: Hypoxia and hepatocellular carcinoma: The therapeutic target for hepatocellular carcinoma. J Gastroenterol Hepatol 22: 1178-1182, 2007.

5. Semenza GL: Hypoxia-inducible factor 1 (HIF-1) pathway. Sci STKE 2007: cm8, 2007.

6. Lee JW, Bae SH, Jeong JW, Kim SH and Kim KW: Hypoxia-inducible factor (HIF-1)alpha: Its protein stability and biological functions. Exp Mol Med 36: 1-12, 2004.

7. Koeppen M,EckleT andEltzschig HK: The hypoxia-inflammation link and potential drug targets. Curr Opin Anaesthesiol 24: 363-369, 2011

8. Beutler B: Tlr4: Central component of the sole mammalian LPS sensor. Curr Opin Immunol 12: 20-26, 2000.

9. Dhupar R, Klune JR, Evankovich J, Cardinal J, Zhang M, Ross M, Murase N, Geller DA, Billiar TR and Tsung A: Interferon regulatory factor 1 mediates acetylation and release of high mobility group box 1 from hepatocytes during murine liver ischemia-reperfusion injury. Shock 35: 293-301, 2011.

10. Cario E and Podolsky DK: Differential alteration in intestinal epithelial cell expression of Toll-like receptor 3 (TLR3) and TLR4 in inflammatory bowel disease. Infect Immun 68 : 7010-7017, 2000

11. Edfeldt K, Swedenborg J, Hansson GK and Yan ZQ: Expression of toll-like receptors in human atherosclerotic lesions: A possible pathway for plaque activation. Circulation 105: 1158-1161, 2002.

12. Lall H, Coughlan K and Sumbayev VV: HIF-1alpha protein is an essential factor for protection of myeloid cells against LPS-induced depletion of ATP and apoptosis that supports toll-like receptor 4-mediated production of IL- 6 . Mol Immunol 45: 3045-3049, 2008

13. Peyssonnaux C, Cejudo-Martin P, Doedens A, Zinkernagel AS, Johnson RS and Nizet V: Cutting edge: Essential role of hypoxia inducible factor-1alpha in development of lipopolysaccharide-induced sepsis. J Immunol 178: 7516-7519, 2007.
14. Sumbayev VV: LPS-induced toll-like receptor 4 signalling triggers cross-talk of apoptosis signal-regulating kinase 1 (ASK1) and HIF-1alpha protein. FEBS Lett 582: 319-326, 2008.

15. Matsuzawa A, Saegusa K, Noguchi T, Sadamitsu C, Nishitoh H, Nagai S, Koyasu S, Matsumoto K, Takeda K and Ichijo H: ROS-dependent activation of the TRAF6-ASK1-p38 pathway is selectively required for TLR4-mediated innate immunity. Nat Immunol 6: 587-592, 2005

16. Livak KJ and Schmittgen TD: Analysis of relative gene expression data using real-time quantitative PCR and the 2(-Delta Delta C(T)) Method. Methods 25: 402-408, 2001.

17. Daoud A, Song J, Xiao F and Shang J: B-9-3, a novel $\beta$-carboline derivative exhibits anti-cancer activity via induction of apoptosis and inhibition of cell migration in vitro. Eur J Pharmacol 724: 219-230, 2014.

18. Harris AL: Hypoxia - a key regulatory factor in tumour growth. Nat Rev Cancer 2: 38-47, 2002.

19. Zeng W, Liu P, Pan W, Singh SR and Wei Y: Hypoxia and hypoxia inducible factors in tumor metabolism. Cancer Lett 356 263-267, 2015

20. Semenza GL: HIF-1 mediates metabolic responses to intratumoral hypoxia and oncogenic mutations. J Clin Invest 123: 3664-3671, 2013.

21. Onishi H, Morisaki T, Nakao F, Odate S, Morisaki T and Katano M: Protein-bound polysaccharide decreases invasiveness and proliferation in pancreatic cancer by inhibition of hedgehog signaling and HIF-1 $\alpha$ pathways under hypoxia. Cancer Lett 335: 289-298, 2013

22. Pugh CW and Ratcliffe PJ: Regulation of angiogenesis by hypoxia: Role of the HIF system. Nat Med 9: 677-684, 2003.

23. Wang GL, Jiang BH, Rue EA and Semenza GL: Hypoxia-inducible factor 1 is a basic-helix-loop-helix-PAS heterodimer regulated by cellular $\mathrm{O}_{2}$ tension. Proc Natl Acad Sci USA 92: 5510-5514, 1995

24. Liu H, Xu R, Feng L, Guo W, Cao N, Qian C, Teng P, Wang L, Wu X, Sun Y, et al: A novel chromone derivative with anti-inflammatory property via inhibition of ROS-dependent activation of TRAF6-ASK1-p38 pathway. PloS One 7: e37168, 2012.

25. Grabinski N, Bartkowiak K, Grupp K, Brandt B, Pantel K and Jücker M: Distinct functional roles of Akt isoforms for proliferation, survival, migration and EGF-mediated signalling in lung cancer derived disseminated tumor cells. Cell Signal 23: 1952-1960, 2011

26. Yamaguchi R, Yano H, Iemura A, Ogasawara S, Haramaki M and Kojiro M: Expression of vascular endothelial growth factor in human hepatocellular carcinoma. Hepatology 28: 68-77, 1998.

27. Soufla G, Sifakis S and Spandidos DA: FGF2 transcript levels are positively correlated with EGF and IGF-1 in the malignant endometrium. Cancer Lett 259: 146-155, 2008.

28. Jensen RL, Ragel BT, Whang K and Gillespie D: Inhibition of hypoxia inducible factor- $1 \alpha$ (HIF- $1 \alpha$ ) decreases vascular endothelial growth factor (VEGF) secretion and tumor growth in malignant gliomas. J Neurooncol 78: 233-247, 2006.

29. O'Neill LA and Bowie AG: The family of five: TIR-domain-containing adaptors in toll-like receptor signalling. Nat Rev Immunol 7: 353-364, 2007.

30. Spirig R, Djafarzadeh S, Regueira T, Shaw SG, von Garnier C, Takala J, Jakob SM, Rieben R and Lepper PM: Effects of TLR agonists on the hypoxia-regulated transcription factor HIF-1alpha and dendritic cell maturation under normoxic conditions. PloS One 5: e0010983, 2010. 